was slightly diminished in one (case 3). Intracellular killing by monocytes showed a heterogeneous picture: either the monocytes were hyperactive or defective in this respect. The hyperactive killing might be explained as a compensatory mechanism in chronic candida infection. The slightly decreased killing by monocytes in case 3 was probably also due to diminished phagocytosis as our killing assay measures the overall result of phagocytosis and intracellular killing.

In cases 1 and 2 the killing defect was remarkable because this defect is serum-dependent. The conclusion that only the disturbance of intracellular killing is serum-dependent is justified, because the phagocytosis assay showed no difference between normal and patient's serum. The question of whether the serum of these two patients contains an inhibitor or lacks a stimulant is under investigation. In two patients with CMC an inhibitor of intracellular killing of candida by granulocytes has been reported. ${ }^{3}$

We thank Dr C M R Weemaes (Nijmegen), Dr G Hendrickx and Dr D B de Geer (Utrecht), and Dr R Schuurman (Rotterdam) for referring their patients. This study was supported by the J A Cohen Institute of Radiopathology and Radiation Protection.

1 Valdimarsson, H, et al, Cellular Immunology, 1973, 6, 348.

2 Clark, R A, et al, Annals of Internal Medicine, 1973, 78, 515

3 Chilgren, R A, et al, Federation Proceedings, 1969, 28, 498.

${ }^{4}$ Leijh, P C J, van den Barselaar, M Th, and van Furth, R, Infection and Immunity, 1977, 17, 313.

${ }^{5}$ Lehrer, R I, and Cline, M J, fournal of Bacteriology, 1969, 98, 996.

(Accepted 18 October 1977)

Laboratory for Cellular Immunology, Department of Infectious Diseases, University Hospital, Leiden, The Netherlands

J W M VAN DER MEER, MD, head of outpatient clinic for infectious diseases

P C J LEIJH, BSC, research fellow

$M$ VAN DEN BARSELAAR, technician

$R$ VAN FURTH, MD, FRCPED, professor of internal medicine and infectious diseases

\section{Prophylaxis with deglycyrrhizinised liquorice in patients with healed gastric ulcer}

Recurrence of gastric ulceration after medical treatment is common, with recorded rates of $40 \%^{1}$ and $60 \%{ }^{2}$ in the first two years. The use of ulcer-healing drugs to prevent relapse has received relatively little attention, though carbenoxolone has been used, but with little success. $^{3}$ An acceptable prophylactic drug would be of considerable value in the management of gastric ulceration, and we undertook a trial of deglycyrrhizinised liquorice (DGL) to assess its merit in this respect.

\section{Patients, methods, and results}

Forty-one patients ( 23 men, 18 women) with benign chronic gastric ulceration, in whom complete ulcer healing had been shown both radiologically and endoscopically within the previous four weeks, were selected for this study. All were aged under 75 , and women of child-bearing age were excluded.

The trial was a double-blind controlled study, in which participants received five capsules a day, each containing either $450 \mathrm{mg}$ of DGL (Ulcedal) or an identical placebo. A normal diet was allowed and alcohol and tobacco were permitted in moderation. Antacids were taken as required. We reviewed the patients monthly for recurrence of symptoms, and a full haematological and biochemical profile was taken at each visit. Gastroscopy and barium-mea examinations were performed at six-monthly intervals, or earlier if dyspeptic symptoms arose in the meantime. Patients were followed up for at least two years or until the ulcer recurred.

On completion of the study eight patients had withdrawn, leaving 33 patients for analysis, of whom 22 had received placebo and 11 DGL. The composition of these groups and the ulcer recurrence rate on DGL and placebo is shown in the table. Eighteen patients developed a further gastric ulcer: five were receiving DGL and 13 placebo. This represents a relapse rate during follow-up of $45 \%$ for DGL and $59 \%$ for placebo. This difference
Comparative clinical data (ranges given in parentheses) and ulcer recurrence rate in 33 patients with a healed chronic gastric ulcer treated prophylactically with either deglycyrrhizinised liquorice (DGL) or placebo

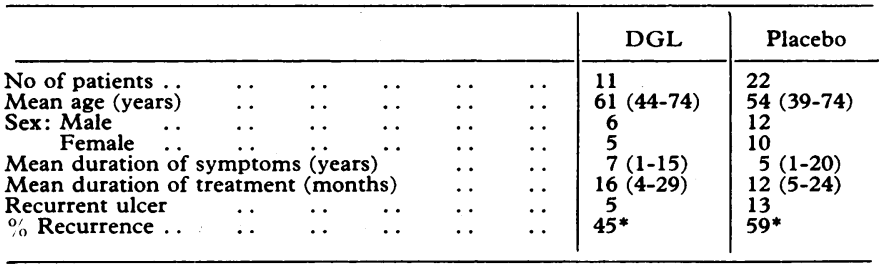

*Difference not significant.

is not significant. Seventy-eight per cent of the total recurrences occurred within the first 10 months of prophylaxis.

Reasons for withdrawal from the trial were defection from follow-up (five patients) and intercurrent illness (three patients). No clinical, bio chemical, or haematological abnormality was detected during treatment and no evidence of long-term toxicity was found.

\section{Comment}

A compound capable of preventing the recurrence of gastric ulceration would have considerable medical, social, and economic importance. Because the mechanism of gastric ulcer recurrence is unknown the choice of a suitable test compound cannot be entirely rational. We chose DGL because of its reputed effectiveness in healing peptic ulceration ${ }^{4}$ (although this has been disputed ${ }^{5}$ ) and its lack of side effects. It has not previously been used prophylactically in man, but has been shown to protect against gastric ulceration in pylorus-ligated rats. DGL in the dosage used in our study is not an effective prophylactic of chronic gastric ulceration, though the recurrence rate was lower in the treated patients than in the placebo group. Nevertheless, the patients' acceptance of treatment combined with regular endoscopy and barium-meal studies was extremely good, which suggests that prolonged treatment and follow-up could be acceptable to informed patients.

Without precise information on the aetiology of gastric ulceration, the choice of further agents as potential prophylactics must be partly intuitive. Indeed, we cannot be certain that ulcer-healing agents will have any pharmacological activity as prophylactics. Nevertheless, we believe that the search for an effective prophylactic should continue, and suggest that the ideal characteristics of such a drug should be infrequent dosage, low cost, lack of side effects, and absence of longterm ill effects, tolerance, or teratogenicity.

We thank Mrs E M McCreery and Miss T M Hughes for typing the manuscript and Boehringer Ingleheim Ltd for supplying active drug and placebo.

1 Veterans Administration Co-operative Study on Gastric Ulcer, Gastroenterology, 1971, 61, No 4, Part 2, 567.

2 Gill, A M, British Medical fournal, 1968, 3, 415.

3 Montgomery, R D, Mehta, S C, and Lawrence, I H, Practitioner, 1969 , $202,398$.

Turpie, A G G, Runcie, J, and Thomson, T J, Gut, 1969, 10, 299.

5 Engqvist, A, et al, Gut, 19.73, 14, 711.

(Accepted 29 November 1977)

Departments of Medicine and Surgery, University Hospital of South Manchester, Manchester M20 8LR

D HOLLANDERS, MSC, MRCP, lecturer in medicine

G GREEN, BM, MRCP, lecturer in medicine (now at the University of Southern California)

I L WOOLF, BM, MRCP, lecturer in medicine (now consultant physician, North Middlesex Hospital, Edmonton)

B E BOYES, MB, MRCP, lecturer in medicine (now consultant physician, Ashton and District General Hospital, Ashton under Lyne)

R Y WILSON, FRCS, lecturer in surgery (now consultant surgeon, North Lonsdale Hospital, Barrow-in-Furness)

D J COWLEY, CHM, FRCS, senior lecturer in surgery (now consultant surgeon, Victoria Hospital, Blackpool)

Department of Gastroenterology, Stepping Hill Hospital, Stockport, Cheshire

I W DYMOCCK, MB, FRCPED, GLAS, consultant physician 